\title{
Clinicopathological correlation of polypoidal choroidal vasculopathy revealed by ultrastructural study
}

\author{
A Okubo, M Sameshima, A Uemura, S Kanda, N Ohba
}

Br J Ophthalmol 2002;86:1093-1098

Aims: To describe the clinical and histopathological findings in a patient with polypoidal choroidal vasculopathy. Methods: A 76 year old Japanese man had a discrete, orange-red lesion of 1 disc diameter in the macula, with the fluorescein and indocyanine green angiographic and optical coherence tomographic findings compatible with polypoidal choroidal vasculopathy. He underwent a surgical removal of the macular lesion, followed by light and electron microscopic examinations.

Results: The histopathological examination revealed that the specimen consisted of degenerated retinal pigment epithelium-Bruch's membrane-choriocapillaris complex and inner choroid. A tortuous, unusually dilated venule was present adjacent to an arteriole with marked sclerotic changes, appearing to form arteriovenous crossing. These vessels seemed to represent native inner choroidal vessels, and had haemorrhage per diapedesis. Blood cells and fibrin filled the lumina of the vessels and accumulated in the extravascular spaces, indicating vascular stasis.

Conclusion: Hyperpermeability and haemorrhage due to stasis of a dilated venule and an arteriole involved by sclerosis at the site where they cross in the inner choroid might cause oedema and degeneration of the tissue. Voluminous accumulation of blood cells and fibrin might generate elevation of tissue pressure sufficient to displace the weakened lesion anteriorly. The result suggests that the polypoidal vessels in this case represent abnormality in the inner choroidal vasculature.

$\mathrm{P}$ olypoidal choroidal vasculopathy (PCV) is a distinct clinical entity characterised by its morphological features ${ }^{1-10}$ aneurysmal dilations seen as reddish-orange, spheroidal, polyp-like structures or polypoidal vascular lesions at the termination of a network of large choroidal vessels. Patients with this disorder are known to be at risk of having recurrent serous and haemorrhagic detachments of both the retinal pigment epithelium (RPE) and the neurosensory retina. This appears to be a common presentation of macular disease in middle and late life in east Asia. ${ }^{8}$ The vascular abnormality appears to exist external to the RPE, but only a few cases have been studied histopathologically ${ }^{11-13}$ and the location and feature of the pathological vessels are controversial. Concerning the origin of the vessels, there are two hypotheses: a variant of choroidal neovascularisation ${ }^{5-13} v$ abnormalities in the inner choroidal vessels. ${ }^{14}{ }^{15}$ This study reports the clinicopathological correlation in a case of PCV whose macular lesion was removed and examined by light and electron microscopy.

\section{PATIENT AND METHODS}

Case report

A 76 year old Japanese man presented with blurred left eye vision for 2 weeks. He lost right eye central vision in the fourth decade of life. He had no systemic disorder. He had not received treatment to either eye. Best corrected visual acuity was 20/200 with the right eye and 20/250 in the left. Fundus examination of the right eye revealed a small atrophic choroidal scar and a few drusen in the posterior pole. In the left eye was a discrete, 1 disc diameter, oval, orange-red subretinal lesion displaying several polypoidal structures and haemorrhagic pigment epithelial detachment in the macula, accompanied by serous retinal detachment and surrounded by numerous subretinal exudative deposits (Fig lA). There were a few drusen in the posterior pole, and round, elongated atrophic choroidal scars inferior to the macular lesion and extending to the equator. Fluorescein angiography of the macular lesion revealed several hyperfluorescent spots with circumferential blocked fluorescence, some of which showed leakage in the late phase of angiography. The indocyanine green (ICG) angiography (IMAGEnet 640, version 1.01; Topcon, Tokyo, Japan) revealed a faint, horseshoe-shaped fluorescence in the early choroidal arteriolar filling phase (Fig 2C), followed by the appearance of a tortuous vascular pattern with aneurysmal or polypoidal structures close to, or overlapping, the horseshoe-shaped vessel, and marked dye leakage (Fig 2D). Optical coherent tomography through the macular lesion demonstrated an anterior bulging of highly reflective layers (Fig 2B).

Over the next 3 weeks extension of the hyperfluorescent areas to the centre of the fovea (Fig 2A) resulted in further decrease of visual acuity. With appropriate informed consent, pars plana vitrectomy and removal of the subretinal lesion was performed by one of the authors, using established techniques. ${ }^{16}$ The postoperative course was uneventful, and the retina reattached with loss of RPE and choriocapillaris of 1 disc diameter area neighbouring, but not involving, the foveola (Fig 1B). No recurrence occurred over the next 2 years. The patient's visual acuity was improved to 30/100 eight months after the surgery and has not deteriorated since.

\section{Methods for preparation of tissue}

The excised tissue, measuring $2.0 \mathrm{~mm} \times 2.0 \mathrm{~mm} \times 0.8 \mathrm{~mm}$, was fixed in phosphate buffered mixture of $2 \%$ paraformaldehyde and $2.5 \%$ glutaraldehyde, postfixed in $2 \%$ phosphate buffered ( $\mathrm{pH}$ 7.4) osmium tetroxide, dehydrated in ethanol series, and embedded in epoxy resin. Semithin serial sections, stained with toluidine blue, were examined by light microscopy. Ultrathin sections, stained with uranyl acetate and lead citrate, were examined using a Hitachi 7100 (Hitachi, Katsuta, Japan) electron microscope.

\section{RESULTS}

Light microscopically (Fig 3), the specimen consisted largely of vascular channels and extravascular blood cells and fibrin, which accumulated particularly in the area posterior to dilated vessels and adhered to the surface of the specimen. A hyalinelike layer occupied the total surface of the specimen. Pigment containing cells were internal (vitreal side) to the hyaline-like 

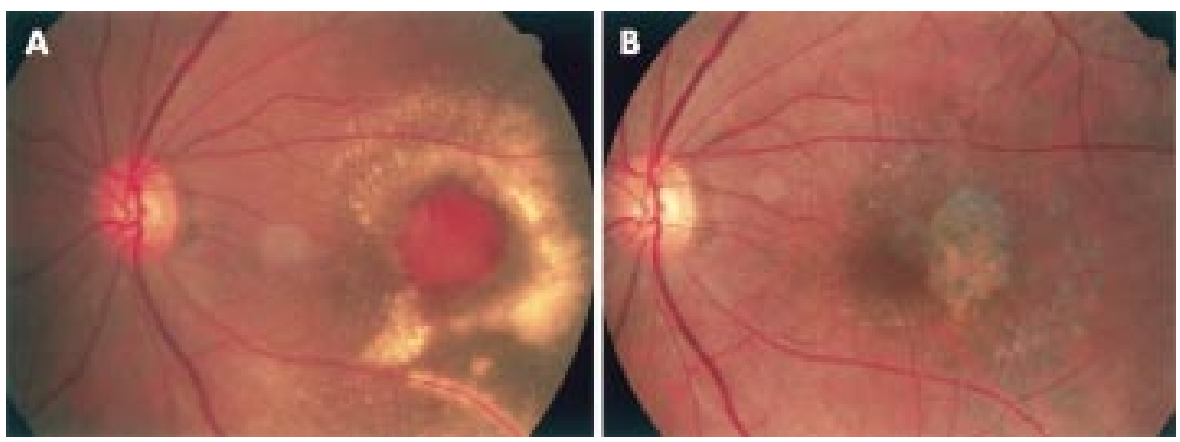

Figure 1 Colour fundus photographs of the left eye. (A) Fundus photograph shows an elevated, oval, 1 disc diameter size, orange-red lesion showing several polypoidal structures in the central portions with an overlying haemorrhagic pigment epithelial detachment and subretinal haemorrhage. There is a ring of exudates surrounding the neurosensory retinal detachment which overlies the lesion. (B) Eight months after operation there is an RPE defect slightly temporal to the foveola.
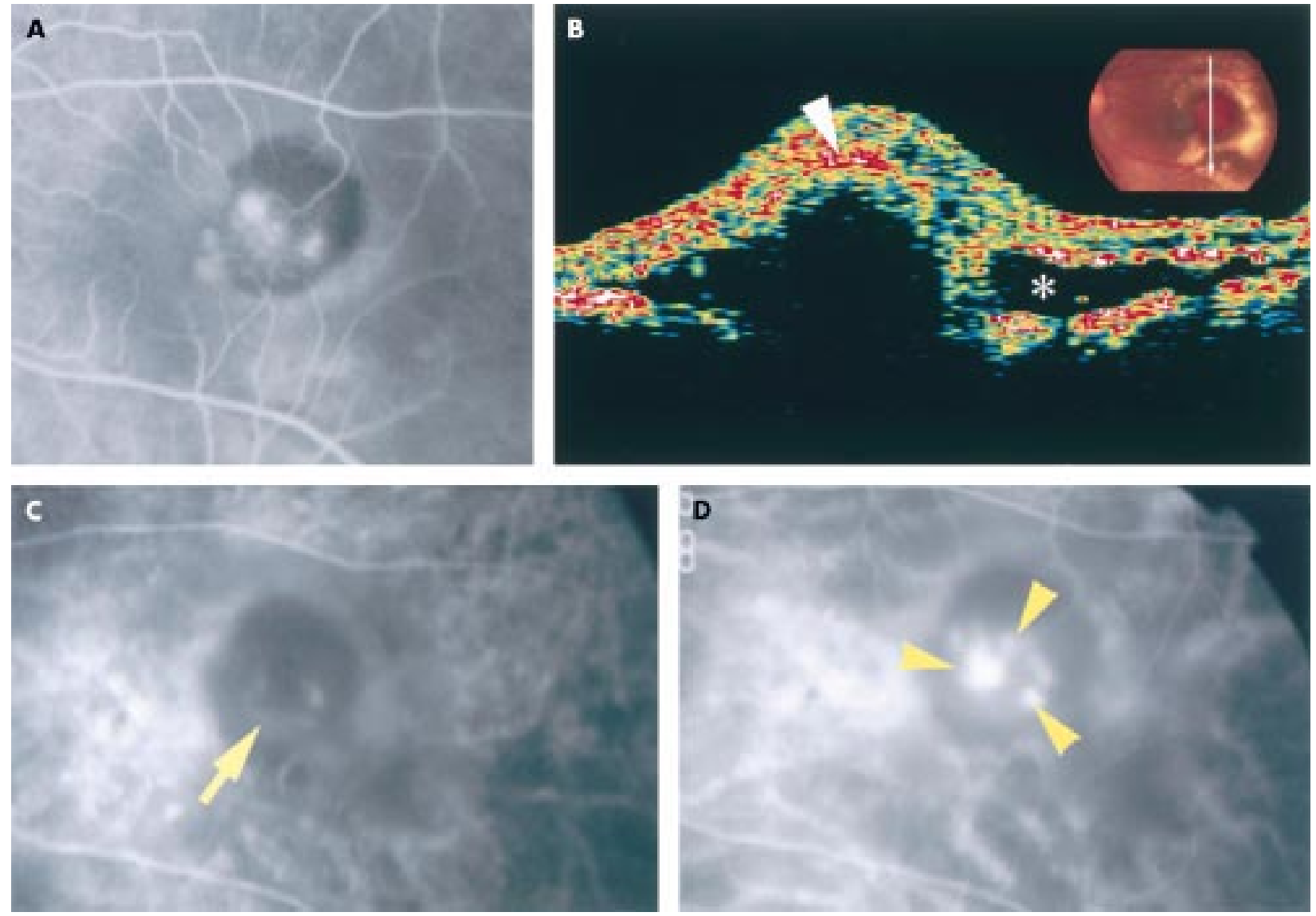

Figure 2 The left eye of the patient. (A) Fluorescein angiogram taken 58 seconds after dye injection shows several hyperfluorescent spots, some of which are leaking, in the macula surrounded by blocked fluorescence. (B) Optical coherence tomographic image scanning the orange-red lesion demonstrates anterior bulging of highly reflective layers which comprise the sensory retinal layer and the surface layer (arrowhead) of the bulged tissue, shadowing the underlying portion. There is a low reflective space (asterisk) suggestive of serous retinal detachment. (C) Indocyanine green angiogram taken 23 seconds after dye injection demonstrates a faint, horseshoe-shaped fluorescence of a large vessel (arrow) in the lesion. (D) Indocyanine green angiogram after 68 seconds. A tortuous vascular structure with polyp-like structures (arrowheads) is seen near, or overlapping with, the horseshoe-shaped vessel.

layer. Variably degenerated capillaries were present along the external (scleral) side of the hyaline-like layer. One or two large venules and arterioles were found externally to the capillaries in each section. The vascular walls of these vessels also appeared hyaline-like. Observations of serial sections suggested that these venules represent one large tortuous venule. Some portions of the venule were sharply bent showing constriction and dilation (Fig 3B). The lumen, as large as $300 \mu \mathrm{m}$, was filled with blood cells and small masses of fibrin. Some of those dilated portions of the venule neighboured an arteriole.
Such an arteriole was also filled with blood cells and fibrin. The venule and arteriole appeared to have formed an arteriovenous crossing (Fig 3A).

Ultrastructurally, the hyaline-like layer on the surface of the specimen appeared to be altered Bruch's membrane combined with thickened basement membranes of vessels that were variably degenerate (Figs 4 and 5). The native choriocapillaris was almost missing at the central surface portion of the lesion, although occasional ghost choriocapillaries were seen which had lost endothelium and pericytes leaving basement 


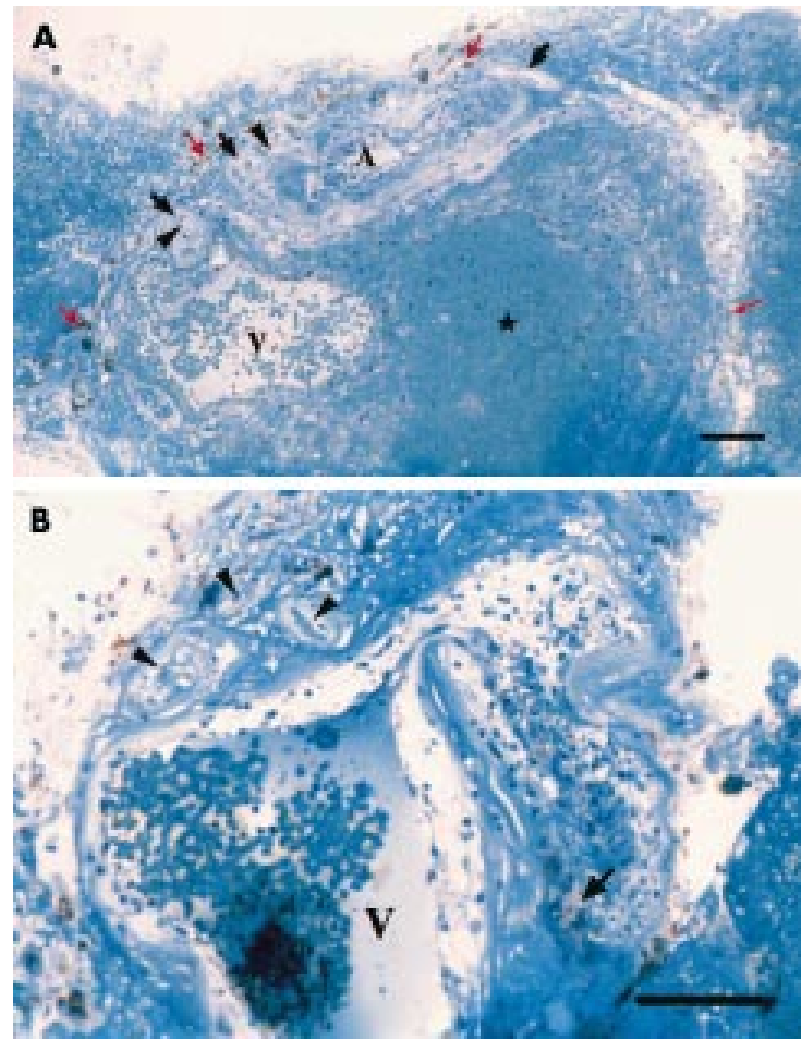

Figure 3 Photomicrographs of approximately vertically sectioned separate portions of the excised specimen (toluidine blue staining). (A) Degenerate pigment containing cells (red arrows), hyaline-like layer (black arrows), and capillaries (arrowheads) occupy its inner surface. A large venule (V) with extravasated blood cells in the wall lies adjacent to an arteriole (A). Blood cells and fibrin are accumulated within, and on the lateral surface of the specimen. Bar $=100 \mu \mathrm{m}$. (B) A tortuous venule (V) shows bent and dilated portions, whose wall is oedematous with extravasated blood cells and lipid laden cells (arrow). The lumen is filled with blood cells and masses of fibrin. Degenerate small arterioles and capillaries (arrowheads) with thickened basement membrane and obstructed lumen lies adjacent to the venule. Bar $=100 \mu \mathrm{m}$

membrane alone (Fig 4). At the peripheral portion of the lesion, the choriocapillaris showed variable aspects nearly normal to degenerative, with thickened basement membrane (Figs 5 and 6C). The pigment containing cells, having melanin and lipofuscin granules, were degenerate RPE cells. They were nearly intact at the peripheral portion (Fig 5). Their basement membranes appearing unusually electron dense formed a continuous sheet even at the sites where some degenerated RPE cells were lost, and were corrugating parallel to the uneven hyaline-like layer (Fig 4). Materials including wide spaced collagen and vesicular and linear profiles were present beneath the degenerate RPE cells and within the innermost portion of the hyaline-like layer (Figs 4 and 5). The outer portion of the hyaline-like layer, studded with remnants of some degenerated cells, was thickened basement membranes of degenerate capillary, arteriole, or venule.

A large arteriole and a few small arterioles were present. The small arterioles showed thickened basement membranes, degenerate smooth muscle cells, degenerate or missing endothelial cells, and narrowed or obstructed lumens (Fig 3). The large arteriole was also degenerate: the smooth muscle cells were variably degenerated (Fig 6A, B); basement membrane substances between the smooth muscle cells increased; the media was frequently undulated; extravasated blood cells were observed in the arteriolar wall, particularly in the edematous spaces surrounding the endothelium; the

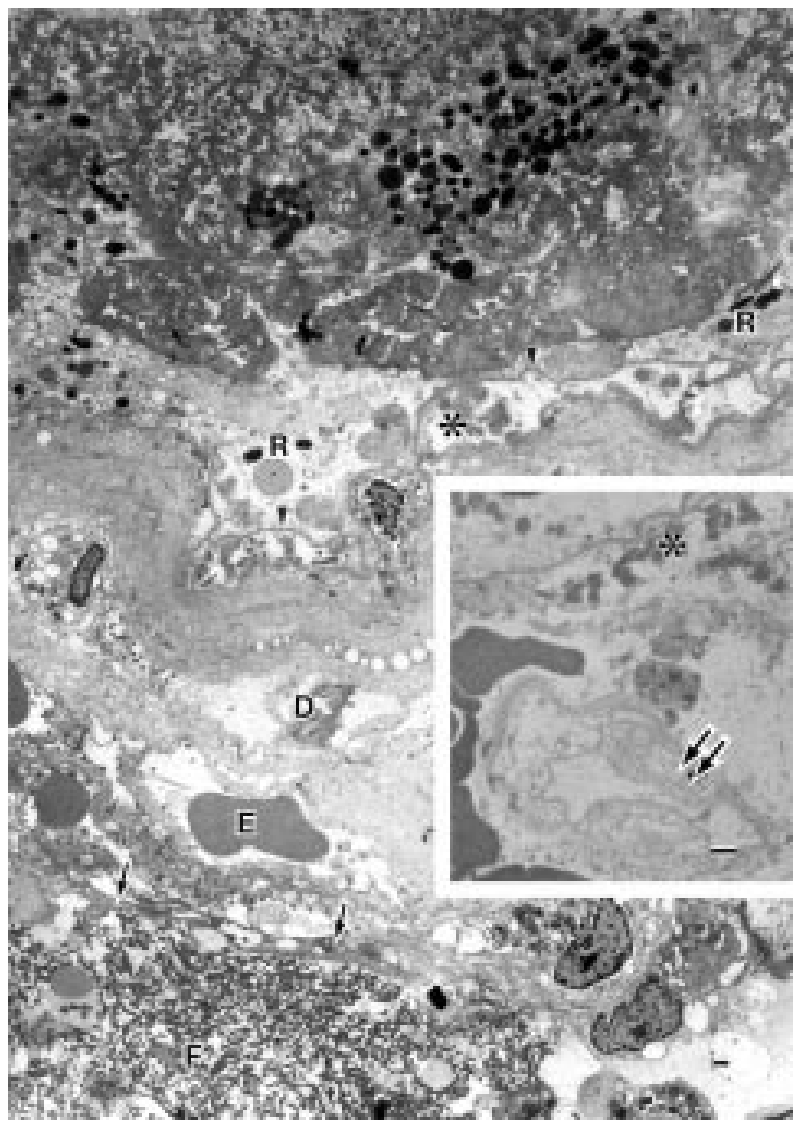

Figure 4 Electron micrograph showing the hyaline-like layer on the surface of the lesion. The basement membrane (arrowheads) of the pigment containing cells $(R)$ is continuous and unusually electron dense. A few collagen fibrils, many wide spaced collagen (asterisk) and vesicular/linear deposits exist beneath the basement membrane. A portion of the vitreal side wall of a large arteriole is seen which is a serial section of the large arteriole $(\mathrm{A}$ ) in Figure $3 \mathrm{~A}$ (a portion of the scleral side wall of this vessel is shown in Fig 6). The arteriole, whose endothelium is indicated by arrows, contains blood cells and fibrin masses $(F)$ in lumen. The seriously degenerated wall consists almost entirely of basement membrane-like substances and remnants of some degenerated cells (D), comprising the outer portions of the hyaline-like layer. Erythrocytes (E) are extravasated in the wall around the endothelium. Inset: A ghost choriocapillary (double arrows) with basement membrane remnant is seen beneath the degenerated Bruch's membrane containing wide spaced collagen (asterisk). Bar $=1 \mu \mathrm{m}$.

lumen was filled with blood cells and fibrin (Fig 3). A largely dilated venule was present adjacent to the large arteriole. Both vessels shared a common adventitia, appearing to be arteriovenous crossing (Fig 3A). The endothelial cells of the dilated venule had frequent intercellular gaps through which erythrocytes escaped (Fig 7). The walls of the venule were oedematous with extravasated blood cells (Fig 7). The extravascular spaces were often oedematous. Numerous blood cells and fibrin masses (Fig 3A), occasional macrophages, fibroblasts, lipid laden cells, and cholesterol slits were present throughout the extra vascular space, particularly accumulated posterior to the dilated vessels.

Capillaries, generally characterised by plump endothelial cells, primitive or narrow lumen, and discontinuous thin basement membrane suggesting newly formed vessels, were observed near the venule (Fig 8). Capillaries were rarely observed within the degenerative wall of the large arteriole. Components of the neural retina were not found in the specimen examined. 


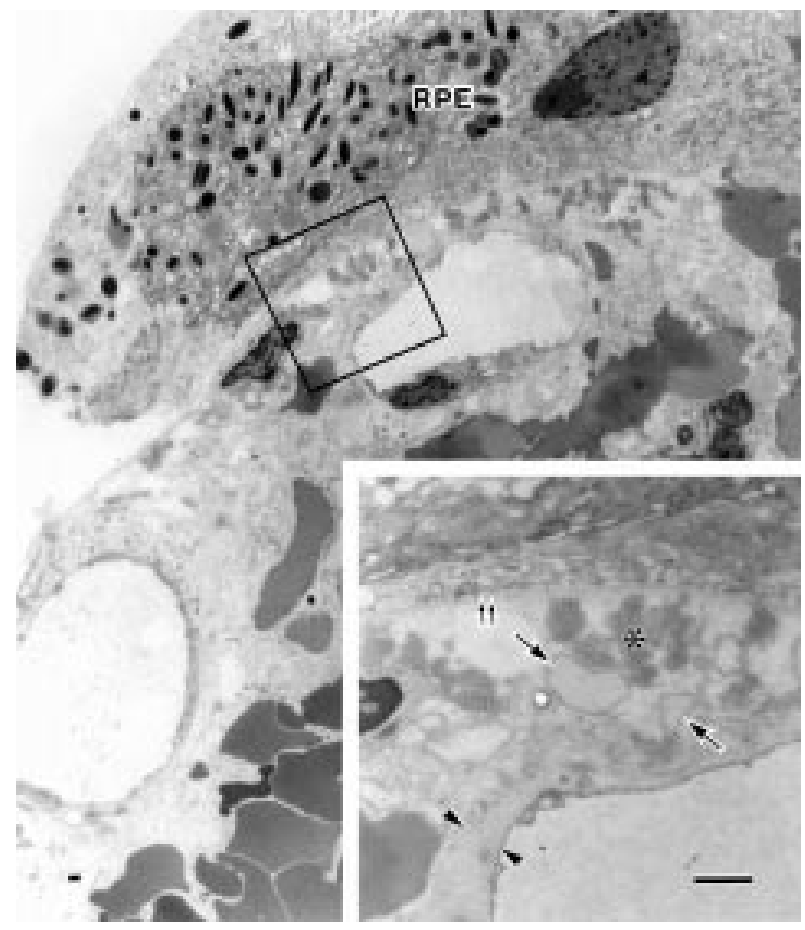

Figure 5 Electron micrograph of a peripheral surface portion of the specimen. The hyaline-like layer is present between the retinal pigment epithelial cells (RPE) and the choriocapillaris that show nearly normal structures. Extravasated erythrocytes are seen mainly external to the choriocapillaris and occasionally in the intercapillary zone. Inset: Magnification of the enclosed area. Bruch's membrane is oedematous and full of wide spaced collagen (asterisk) and vesicular profiles (arrows), showing no normal structures such as elastic layer. Double arrows: Basement membrane of the RPE. The endothelium of the choriocapillaris shows fenestrae and thick basement membrane (arrowheads). Bar $=1 \mu \mathrm{m}$.

\section{DISCUSSION}

The clinical features in this case are consistent with PCV: orange-red polypoidal structures, which showed characteristic features in ICG angiography, associated haemorrhagic RPE and neurosensory retinal detachment, and lipid deposits under the retina. ${ }^{1-8}$

A few clinicopathological studies of PCV have been reported. ${ }^{11-13}$ MacCumber et al ${ }^{10}$ examined an enucleated eye with multiple recurrent serosanguineous retinal pigment epithelial detachments syndrome and disclosed extensive fibrovascular proliferation within Bruch's membrane and in the subretinal space, reflecting that the eye had probably reached an end stage of the disease. They did not observe choroidal varices or aneurysmal dilation of choroidal vessels except that some choroidal veins were quite large. Spraul et al, ${ }^{12}$ examining enucleated eye displaying choroidal vascular-like bulbous structures, described a sub-RPE, intra-Bruch's fibrovascular membrane without a subretinal component but did not note aneurysmal vessels. Lafaut et $a l^{13}$ reported light microscopic findings in the submacular tissue removed from an eye with PCV in age related macular degeneration, describing a sub-RPE, intra-Bruch's fibrovascular membrane that contained several dilated thin walled vessels, a maximal diameter of $140 \mu \mathrm{m}$, under diffuse drusen. The authors noted that the aneurysmal vessels appeared to be of venular origin and were probably the pathological counterpart of the lesion identified in ICG angiography. All these reports described an intraBruch's fibrovascular membrane that might correspond to the clinically observed lesion. The lesion in our case consisted of degenerated RPE-Bruch's membrane-choriocapillaris complex and inner choroid containing largely dilated venules and arterioles.
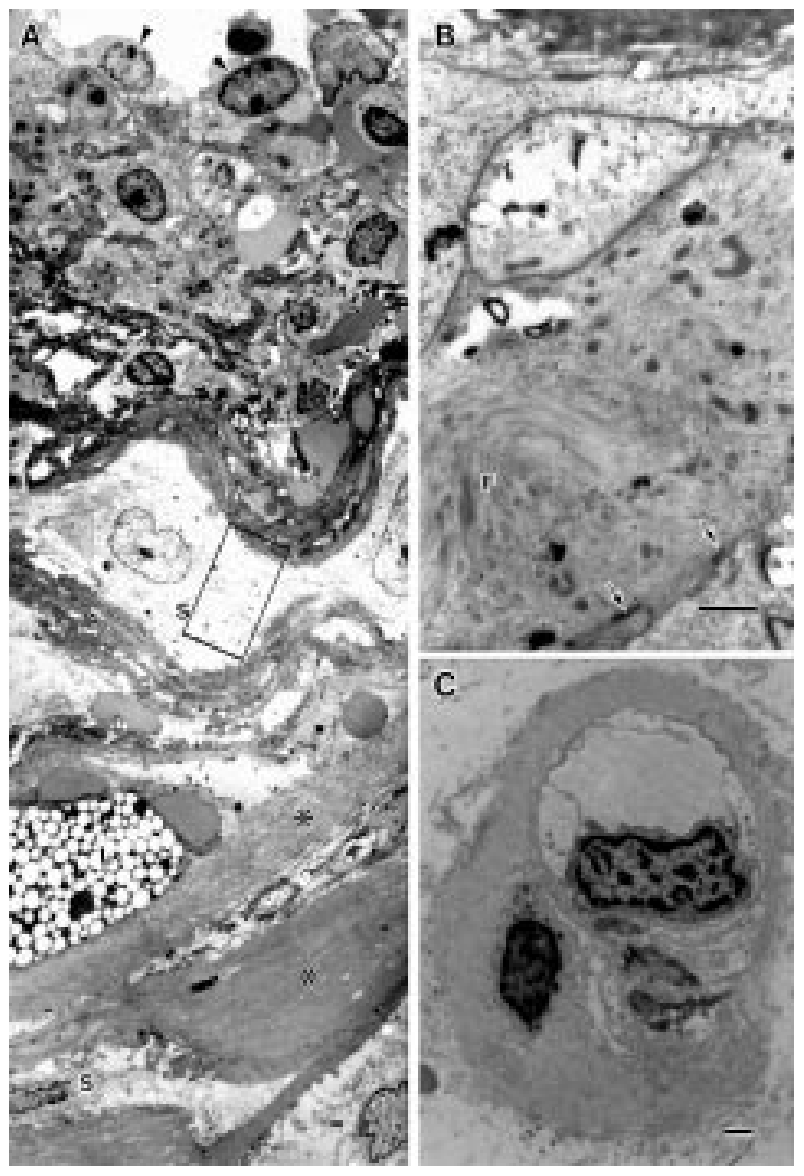

Figure 6 Electron micrographs of a degenerate arteriole and a capillary. (A) A portion of the scleral side wall of the degenerate large arteriole, showing platelets and erythrocytes seen around its endothelial cells (arrowheads), increased basement membrane substances (asterisks), variably degenerate smooth muscle cells (S), and lipid laden cell (L). (B) Magnification of the enclosed area in (A), showing smooth muscle cells with myofilaments (F) and sublemmal dense bodies (arrows). (C) A choriocapillaris with decreased fenestrae and thickened basement membrane. Bar $=1 \mu \mathrm{m}$.

The hyaline-like layer lying continuously on the inner surface of the excised lesion with degenerated RPE cells, at least innermost portion of it, was identified as disorganised degenerated Bruch's membrane. The continuous basement membrane observed beneath the discontinuous layer of degenerated RPE cells probably constitutes the continuous inner aspect of Bruch's membrane. The wide spaced collagen and vesicular and linear profiles (basal laminar deposit and/or basal linear deposit) were observed beneath the degenerated RPE and within the Bruch's membrane, showing that the layer of RPE is native. ${ }^{17}$ The outer portions of the hyaline-like layer were composed mainly of either basement membrane-like substance containing remnants of choriocapillaris or basement membrane-like substance of the degenerated walls of arterioles/venules that constituted the lesion and seemed to be choroidal native, but not new, vessels, as discussed below. Thus, we may regard the lesion as degenerated RPE-Bruch's membrane-choriocapillaris complex and inner choroid rather than an intra-Bruch's fibrovascular membrane.

Noticeable is the finding of a large tortuous venule with several dilated portions, some of which appeared to form arteriovenous crossing with a relatively large arteriole. This is consistent with the vascular pattern seen on the ICG angiography. The endothelial gaps seen in the dilated portions of the venule allowing even erythrocytes to pass out of the vessel indicate haemorrhage per diapedesis. The extravasated blood cells and 


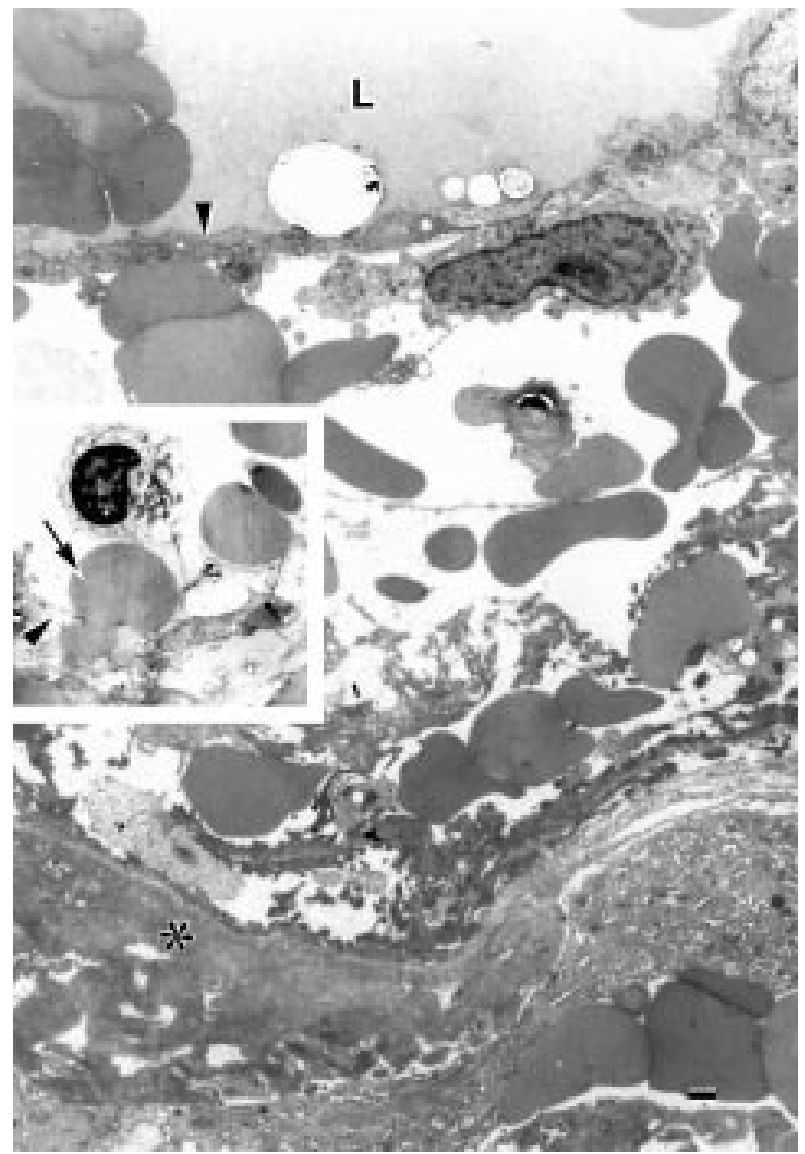

Figure 7 Electron micrograph of a portion of the degenerate wall of a dilated large venule. The wall is oedematous with extravasated erythrocytes and fibrin around endothelium (arrowhead). $\mathrm{L}=$ lumen; asterisk = basement membrane substances. Inset: an erythrocyte (arrow) is passing through a gap between endothelial cells (arrowhead). Bar $=1 \mu \mathrm{m}$.

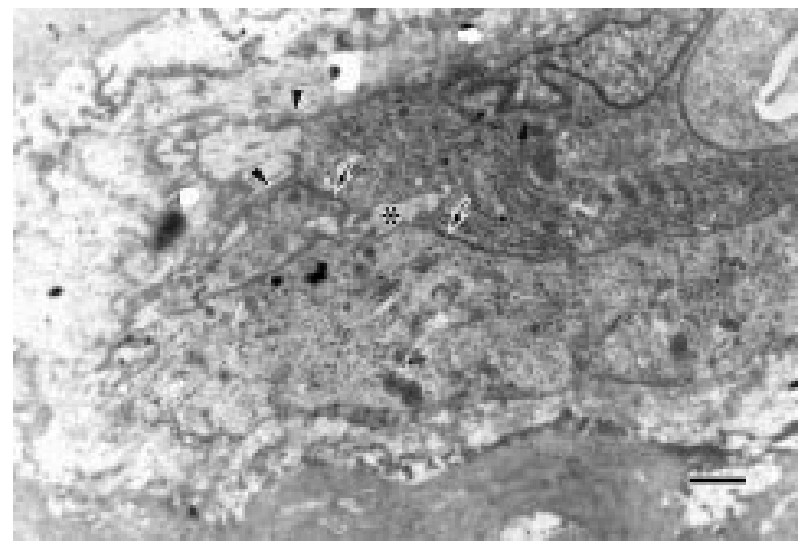

Figure 8 Electron micrograph of a newly formed vessel located near the large dilated venule. The plump endothelial cells with discontinuous thin basement membranes (arrowheads) are bound each other by immature intercellular junction (arrows), and have formed immature lumen (asterisk). Bar $=1 \mu \mathrm{m}$.

fibrinous fluid in the arterial wall also suggest haemorrhage per diapedesis. These findings explain leakage of dye within the PCV lesion in the fluorescein and ICG angiography. The polypoidal structures observed clinically seem to represent the dilated portions of the tortuous venule. The orange-reddish colour of the macular lesion can be attributed to abundant erythrocytes in the dilated large vessels and in extravascular spaces. The diameter as large as $300 \mu \mathrm{m}$, the structures, and the location of the tortuous venule associated with arterioles suggest that it is a native, dilated, choroidal venule rather than a new vessel. Volumes of blood cells and fibrin masses in the dilated lumen and within the wall of this venule suggest that the tortuous venule was involved by stasis. These findings support the views that PCV is degenerative changes or abnormalities in choroidal vessels on the basis of clinical finding $\mathrm{s}^{14}{ }^{15}$ and is perhaps consistent with the clinical findings that polypoidal structures overlying or evaginating directly from the larger underlying choroidal vessels. ${ }^{5}$ On the other hand, Lafaut et $a l^{13}$ observed dilated thin walled vessels (a maximal diameter of $140 \mu \mathrm{m}$ ) appearing to be from venular rather than arteriolar origin, within the intra-Bruch's fibrovascular membrane and ascribed the clinically visible lesions to those dilated vessels. This view probably supports the clinical findings that polypoidal structures resemble fronds of neovascularisation with polyp-like termination. ${ }^{69}$ Apart from the cause of aneurysmal dilation of new vessels in fibrovascular membrane, the dilated vessels, whether native or new vessels, are probably the pathological counterpart of the polypoidal structures identified clinically. A variety of histopathological aspect of PCV may be reasonable in the light of the expanding clinical spectrum of PCV. ${ }^{1-15}$

The question arises as to what caused such dilation of choroidal venules as seen in the present case. In the retina, branch retinal vein occlusion (BRVO) occurs at the arteriovenous crossing portion, particularly in arteriolar sclerosis. ${ }^{18-22}$ In that case, the retinal branch vein is occluded and dilated with degenerative endothelium, extravasated red cells in the vascular wall, and oedema fluid around the occluded vessel. The artery is also involved by thrombosis with lamellar arrangement of basement membrane substances, and atrophic smooth muscle cells and fibrous increase with resultant hyaline-like alteration of vessel walls. Haemorrhage per diapedesis from occluded venules causes arterioles and capillaries to degenerate. ${ }^{19}{ }^{20}$ The histopathological features of the venule and arteriole in the present case resemble those of BRVO. In the choroid, more numerous arterioles and venules are present within the macular and peripapillary areas ${ }^{23-28}$; the venules are tortuous and frequently cross with the arterioles. It is possible that choroidal vasculature may also be involved by stasis of venules by the mechanism similar to that of BRVO. Such a venule affected by stasis may become so fragile that it changes into beaded and polypoidal configuration. Thus, we propose that PCV at least in the present case might be caused by stasis of venule and arteriole at the arteriovenous crossing associated with arteriolar sclerosis. Polypoidal vessels in PCV in some cases are reported to show pulsation on ICG angiography. It may reflect the involvement of arteries.

The hyperpermeability and haemorrhage due to the stasis of vessels might cause oedema and degeneration of the tissue. Concomitantly, the voluminous accumulation of blood cells and fibrin in extravascular spaces, together with the dilated venule and possibly arteriole in the inner choroid, might generate elevation of tissue pressure sufficient to displace the lesion. This view is deduced from the findings in this case: degeneration of Bruch's membrane such as loss of elastic layer, the choriocapillaris disappearing leaving only some remnants, arterioles and venules coming close to altered Bruch's membrane in places, and voluminous accumulation of blood cells and fibrin in extravascular spaces. In the peripheral portion of the lesion where there were extravasated blood cells probably derived from the venules or arterioles involved with stasis, Bruch's membrane was altered in comparison with the nearly intact RPE cells and choriocapillaris, suggesting susceptibility of Bruch's membrane that may allow the lesion to bulge.

Newly formed capillaries were observed within the wall of the degenerate arteriole and near the dilated venule in the present case. Neovascularisation for recanalisation and collateral circulation is known to occur in the ischaemic portions 
associated with BRVO. ${ }^{21} 22$ The pathological features in the present case are similar to those in BRVO. Therefore, the newly formed capillaries for recanalisation and collateral circulation could occur. It is known that the area involved with polypoidal structures shows some type of resolution or regression and is ultimately replaced with a new network of vessels. ${ }^{5}$

We were able to resect the lesion successfully for the following reasons: (1) the RPE, choriocapillaris, and inner choroid comprising the lesion were considerably degenerated, indicating decreased blood circulation; the lesion was localised and bulged into the subretinal space. These conditions might allow us to grasp and remove the lesion by using forceps with less bleeding and without damaging the surrounding tissue, particularly the foveola. (2) The abnormal tissue had not involved the foveola, and the direct cause of the sudden decrease of visual acuity before operation was the blood and/or exudates extending over the foveola. This condition might be favourable for improvement of visual acuity after operation. However, we must keep in mind that surgical treatment of this condition could be associated with a resultant removal of the native RPE and choriocapillaris from the excised area.

In conclusion, ultrastructural examination of the submacular, subretinally bulging tissue surgically removed from an eye of a Japanese elderly patient with PCV revealed that the lesion consisted of degenerated RPE-Bruch's membranechoriocapillaris complex and inner choroid which contained a tortuous, aneurysmally dilated, probable native venule, degenerated arterioles, and voluminous blood cells and fibrin in the extravascular spaces. The dilated venule manifested hyperpermeability and haemorrhage probably as a result of stasis that was probably caused by the venule crossing the arteriole, and was concluded to correspond to polypoidal structures. The voluminous blood /fibrin in the extravascular spaces might cause oedema and degeneration of the tissue and might generate elevation of sufficient tissue pressure for the lesion to bulge subretinally. These results suggest that there is a variety in the histopathological aspect of PCV, such as abnormalities in choroidal vasculature and intra-Bruch's fibrovascular membrane. The condition in this case seems favourable for surgical treatment with a resultant improvement of visual acuity.

\section{ACKNOWLEDGEMENTS}

The authors wish to thank Professors Alan C Bird and Phillip J Luthert, Moorfields Eye Hospital and Institute of Ophthalmology, for reading the manuscript and making a number of helpful suggestions.

\section{Authors' affiliations}

A Okubo, M Sameshima, A Uemura, S Kanda, N Ohba, Department of Ophthalmology, Kagoshima University Faculty of Medicine, Sakuragaoka 8-35-1, Kagoshima 890-8520, Japan

Correspondence to: Akiko Okubo, Kagoshima University Faculty of Medicine, Sakuragaoka 8-35-1, Kagoshima 890-8520, Japan; akiko@m2.kufm.kagoshima-u.ac.jp

Accepted for publication 2 May 2002

\section{REFERENCES}

1 Stern RM, Zakov ZN, Zegarra H, et al. Multiple recurrent serosanguineous retinal pigment epithelial detachments in black woman Am J Ophthalmol 1985;100:560-9.
2 Yannuzzi LA, Sorenson J, Spaide RF, et al. Idiopathic choroidal vasculopathy (IPCV). Retina 1990;10:1-8

3 Kleiner RC, Brucker AJ, Johnston RL. The posterior uveal bleeding syndrome. Retina 1990;10:9-17.

4 Perkovich BT, Zakov ZN, Berlin LA, et al. An update on multiple recurrent serosanguineous retinal pigment epithelial detachments in black woman. Retina 1990;10:18-26.

5 Spaide RF, Yannuzzi LA, Slakter JS, et al. Indocyanine green videoangiography of idiopathic polypoidal choroidal vasculopathy. Retina 1995; 15:100-10.

6 Yannuzzi LA, Ciardella A, Spaide RF. The expanding clinical spectrum of idiopathic polypoidal choroidal vasculopathy. Arch Ophthalmol 1997; 1 15:478-85.

7 Moorthy RS, Lyon AT, Rabb MF, et al. Idiopathic polypoidal choroidal vasculopathy of the macula. Ophthalmology. 1998;105:1380-5.

8 Uyama M, Matsubara T, Fukushima I, et al. Idiopathic polypoidal choroidal vasculopathy in Japanese patients. Arch Ophthalmol 1999:117:1035-42.

9 Yannuzzi LA, Wong DWK, Sforzolini BS, et al. Polypoidal choroidal vasculopathy and neovascularized age-related macular degeneration. Arch Ophthalmol 1999:117:1503-10.

10 Ahuja RM, Stanga PE, Vingerling JR, et al. Polypoidal choroidal vasculopahty in exudative and haemorrhagic pigment epithelial detachments. Br J Ophthalmol 2000;84;479-84.

11 MacCumber MW, Dastgheib K, Bressler NM, et al. Clinicopathologic correlation of the multiple recurrent serosanguineous retinal pigment epithelial detachments syndrome. Retina 1994;14:143-52.

12 Spraul CW, Grossniklaus HE, Lang GK. Idiophathische polypöse choroidale vaskulopathie (IPCV). Klin Monatsbl Augenheilkdd 1997;210:405-6.

13 Lafaut BA, Aisenbrey S, van den Broecke C, et al. Polypoidal choroidal vasculopathy pattern in age-related macular degeneration. A clinicopathologic correlation. Retina 2000;20:650-4.

14 Hayashi K. Clinical application of indocyanine green angiography-pathologic features of idiopathic polypoidal choroidal vasculopathy and congestion choroidopathy. (Abstract in English.) Folia Ophthalmol Jap 1996;47:613-20

15 Akita J, Kimura T, Ojima T, et al. Idiopathic polypoidal choroidal vasculopathy without hemorrhage. Differentiation from central serous chorioretinopathy (Abstract in English.) Jpn Rev Clin Ophthalmol 2001;95:702-7.

16 Thomas MA, Dickinson JD, Melberg NS, et al. Visual results after surgical removal of subfoveal choroidal neovascular membranes. Ophthalmology 1994;101:1384-96.

17 Gass JMD. Biomicroscopic and histopathologic considerations regarding the feasibility of surgical excision of subfoveal neovascular membranes. Am J Ophthalmol 1994;118:285-98.

18 Inomata H, Nomura T. Fine structures of human retinal vessels and their pathological alterations in vascular diseases. (Abstract in English.) Folia Ophthalmol Jap 1971;10:849-58.

19 Kimura T. Arteriolar sclerosis of the human retina. Eye, Ear, Nose and Throat Monthly 1974;53:99-107.

20 Kimura T. Arteriolar sclerosis of the human retina. Eye, Ear, Nose and Throat Monthly 1974;53:134-7.

21 Frangieh GT, Green WR, Barraquer-Somers E, et al. Histopathologic study of nine branch retinal vein occlusions. Arch Ophthalmol 1982;100:1132-40.

22 Bowers DK, Finkelstein D, Wolff SM, et al. Branch retinal vein occlusion. A clinicopathologic case report. Retina 1987;7:252-9.

23 Ashton N. Communications. Observations on the choroidal circulation. Br J Ophthalmol 1952;36;465-81.

24 Wyber KC. A study of the choroidal circulation of the eye in man. $J$ Anatomy 1954;88:94-101.

25 Ring HG, Fujino T. Observations on the anatomy and pathology of the choroidal vasculature. Arch Ophthalmol 1967;78:431-44.

26 Uyama M. Ohkuma H, Itotagawa S, et al. Pathology of choroidal circulatory disturbance. Part 1. Angioarchitecture of the choroid, observation on plastic cast preparation. (Abstract in English.) J Jpn Ophthalmol Soc 1980;84:1893-909.

27 Yoneya S, Tso MOM. Angioarchitecture of the human choroid. Arch Ophthalmol 1987; 105:681-7.

28 Fryczkowski AW. In: Yannuzzi LA, Flower RW, Slakter JS, eds. The choriocapillaris: anatomic and functional choroidal lobules. Indocyanine green angiography. St Louis: CV Mosby, 1997:29-35. 